\title{
Caracterización de sensor de material particulado de bajo costo
}

\section{Characterization of a low-cost particulate matter sensor}

Presentación: 10/08/2021

Aprobación: 18/10/2021

\author{
Ariel Scagliotti \\ Instituto de Ciencias - Universidad Nacional de General Sarmiento - Argentina \\ ascagliotti@campus.ungs.edu.ar
}

\section{Guillermo Jorge}

Instituto de Ciencias - Universidad Nacional de General Sarmiento - Argentina

gjorge@campus.ungs.edu.ar

\section{Resumen}

Las partículas en el aire son un indicador importante sobre la calidad del aire, con probados efectos negativos sobre la salud. A nivel de superficie, se caracterizan como material particulado y su medición bajo norma es escasa o inexistente en países de recursos limitados. Es por ello que los sensores de bajo costo representan una alternativa valiosa para adquirir este tipo de datos ambientales, pero poseen algunas limitaciones que deben ser debidamente caracterizadas antes de su uso. Se presenta un estudio de indicadores de desempeño de un sensor óptico de bajo costo de material particulado, a partir de pruebas de laboratorio, simulaciones con un enfoque físico y comparaciones con un equipo calibrado. Se halló información valiosa sobre las limitaciones del sensor y se desarrolló un código de control para advertir al usuario sobre datos sospechosos.

Palabras claves: sensor, bajo costo, material particulado, caracterización

\begin{abstract}
Particulates in the air are an important indicator of air quality, with proven adverse health effects. At the surface level, they are characterized as particulate matter and their regulatory measurement is scarce or non-existent in countries with limited resources. That is why low-cost sensors represent a valuable alternative to acquiring this type of environmental data, but they have some limitations that must be properly characterized before use. A study of performance indicators of a low-cost optical sensor for particulate matter is presented, based on laboratory tests, simulations with a physical approach, and comparisons with calibrated equipment. Valuable information on sensor limitations was found and a control code was developed to warn the user of suspicious data.
\end{abstract}

Keywords: sensor, low-cost, particulate matter, characterization 


\section{Introducción}

La calidad del aire (CA) es uno de los mayores problemas ambientales mundiales de la actualidad, pues su deterioro está ligado a mortalidad prematura, especialmente en poblaciones vulnerables (Pascal et al., 2013). Uno de los principales indicadores de la CA es el material particulado (PM) que, de acuerdo a su diámetro aerodinámico equivalente, se suele categorizar como PM10 (tamaños menores a $10 \mu \mathrm{m}$ ), PM2.5 (tamaños menores a 2,5 $\mu \mathrm{m}$ ) y PM1 (partículas de tamaños menores a $1 \mu \mathrm{m}$ ).

Medir bajo regulación los contaminantes como el PM requiere de instrumentos muy costosos y equipos de profesionales para mantenerlos y operarlos. Estas condiciones y requerimientos derivan en que se cuente en muchas ciudades del mundo, especialmente en aquellas con bajos recursos, con una cobertura limitada y una distribución irregular de estaciones de monitoreo (Tian et al., 2013). Además, fuera de las grandes urbes, en zonas conurbanas o interiores de los países, no se cuenta generalmente con ningún tipo de medición.

Una alternativa viene dada por los sensores de bajo costo (LCS) para el monitoreo de la CA, utilizados actualmente por iniciativas de ciencia ciudadana, investigación científica y autoridades públicas (Kumar et al., 2015). Los LCS poseen una versatilidad que permite al usuario adaptarlos a proyectos específicos, y son capaces de proporcionar mediciones en tiempo real con una alta resolución temporal abarcando en su espectro efectos debidos a eventos de corta duración (Karagulian et al., 2019). En los últimos años, numerosos trabajos se han dedicado a estimar el desempeño y limitaciones de LCS de CA con resultados variables dependiendo del uso específico (Alfano et al., 2020). Un punto en contra de esta tecnología es que aún no cuenta con protocolos de calibración, validación y evaluación de desempeño exhaustivos que sean universalmente aceptados, aunque existen numerosos esfuerzos al respecto (ver por ejemplo AQ-SPEC 2021). En este sentido hay dos enfoques complementarios, el de gestión automática de calidad de los datos (QA) y el de control de calidad (QC). QA está conformado por procesos o etapas que se aseguran de minimizar las inexactitudes de los datos y la necesidad de medidas correctivas posteriores, y se llevan a cabo de forma automática mientras se están registrando los datos. QC es un proceso que se implementa con los datos ya registrados y comprueba que cumplan con los requisitos de calidad determinados por el usuario (Campbell et al., 2013). La variable interferente más significativa observada con los LCS de PM es la humedad relativa (HR), debido al crecimiento higroscópico que experimentan las partículas en el aire y por verse comprometido el detector (Crilley et al., 2018).

Las técnicas de medición de PM de bajo costo se basan comúnmente en sistemas ópticos que hacen uso de una fuente de luz de baja potencia. Las partículas que ingresan al sensor por el canal de muestreo dispersan la luz que es medida por un fotodetector en el dispositivo. Este método utiliza la teoría de Mie que predice un patrón de dispersión en base a la relación entre la intensidad de la luz y el ángulo de detección, a partir del cual puede determinarse una distribución de tamaños de partículas (Mie, 1976). Con el fin de modelar la distribución de aerosoles presentes en una muestra de aire, se la puede considerar como compuesta por una suma de $\mathrm{n}$ modos lognormales (Seinfeld y Pandis, 2016): 


$$
\frac{d N}{d \log \left(D_{p}\right)}=\sum_{i=1}^{n} \frac{N_{i}}{\sqrt{2 \pi} \log \left(\sigma_{i}\right)} \exp \left(-\frac{\left(\log \left(D_{p}\right)-\log \left(\bar{D}_{p, i}\right)\right)^{2}}{2 \log ^{2}\left(\sigma_{i}\right)}\right)
$$

Donde $\mathrm{N}$ es el número de partículas (en particular, se denomina Ni al número de concentración de cada modo $i$, es decir número de partículas por $\left.\mathrm{cm}^{3}\right), \sigma_{i}$ es la desviación estándar geométrica (variedad del tamaño de partícula) y $\bar{D}_{p, i}$ es la media geométrica del diámetro de partícula (la mediana de la distribución de tamaños para cada modo).

A su vez, se deben determinar algunas características que afectan a las propiedades ópticas de aerosoles para terminar de definir un modo de distribución de partículas. Estas son: el factor higroscópico de crecimiento $(f(\mathrm{RH}))$, la densidad de partículas $(\rho)$ y el índice complejo de refracción $\left(m_{i}\right)$ (cuya parte real indica la razón entre la velocidad de la luz en el medio y en el vacío, y la imaginaria corresponde al coeficiente de extinción de la luz). La función de distribución de probabilidades está dada por la suma final de todos los modos en la Ec. (1), y se denomina también función de número de concentración. Al multiplicarse la distribución de la Ec. (1) por el volumen de partícula simplificado a una esfera se obtiene la distribución volumétrica. Si se integra esta distribución entre dos tamaños de partículas se consigue la función de distribución acumulada volumétrica, y su producto por la densidad permite estimar la carga másica de aerosoles por unidad de volumen, que llamamos PM (en $\left.\mu \mathrm{g} / \mathrm{m}^{3}\right)$, entre los diámetros elegidos (Ec. (2)). Por ejemplo, $\mathrm{PM}_{2.5}$ surge de integrar la distribución volumétrica entre los diámetros $0 \mathrm{y}$ $2,5 \mu \mathrm{m}$, y multiplicar por la densidad $\rho$.

$$
P M=\rho \frac{\pi}{6} \int_{D_{\min }}^{D_{\max }} D_{p}^{3} n_{N}\left(D_{p}\right) d D_{p}
$$

La sede central de la Universidad Nacional de General Sarmiento (UNGS), donde se realizaron los desarrollos y mediciones para este trabajo, se encuentra en una zona sin cobertura histórica de mediciones de CA, en el Centro-Norte del Área Metropolitana de Buenos Aires. Recientemente, se comenzaron a realizar mediciones de propiedades ópticas de aerosoles en la zona (Scagliotti y Jorge, 2020a) y este trabajo presenta un desarrollo para incorporar mediciones de PM en superficie. Se presenta la caracterización de un LCS de PM PMS5003 (PMS de ahora en más) a partir de pruebas de laboratorio, comparaciones con equipo calibrado y simulaciones desde un enfoque físico. De esta manera, se determinan indicadores de desempeño a partir de los cuales se propone un algoritmo para el control de calidad automática (QA) de las mediciones.

\section{Desarrollo}

Se adaptó una cámara de prueba de sensores desarrollada previamente (Scagliotti y Jorge, 2020b) de acuerdo al diseño mostrado en la Fig. 1. 


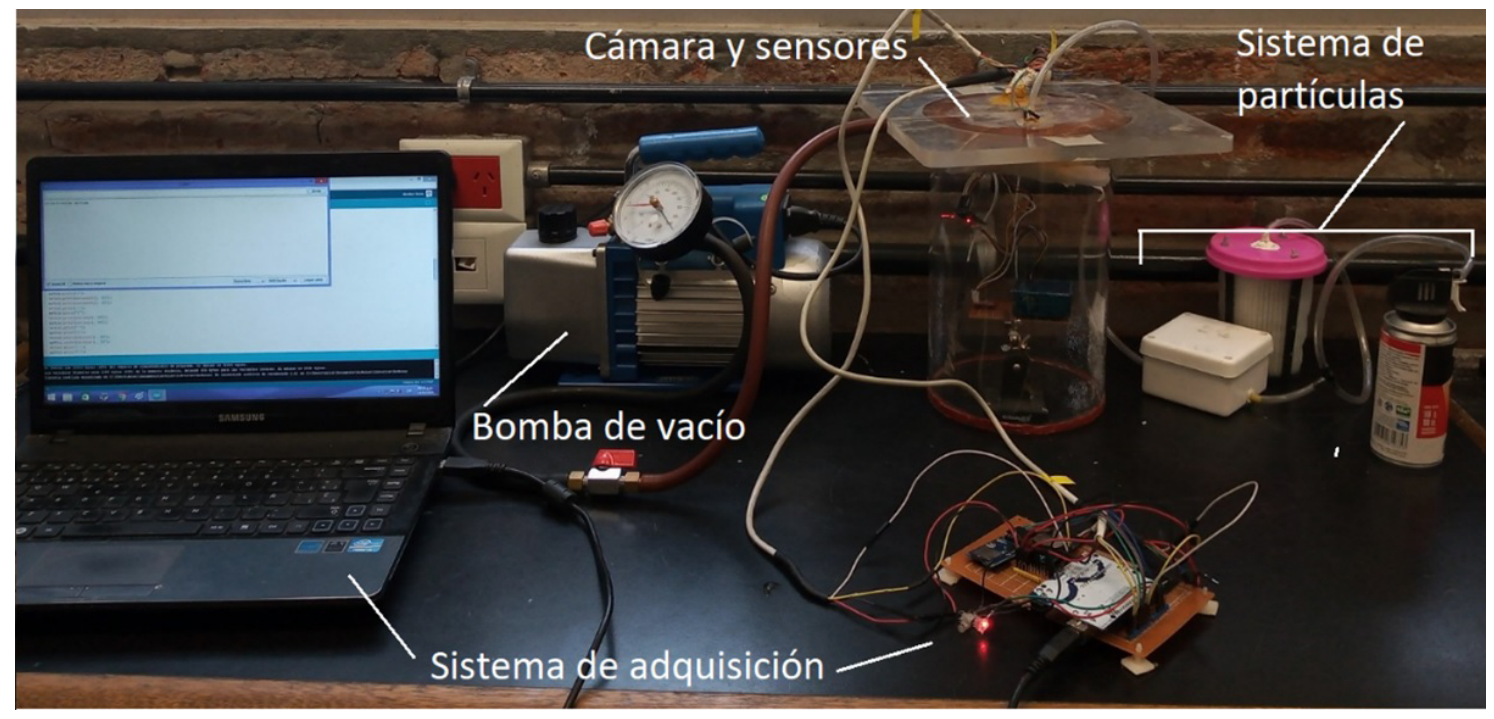

Fig. 1: Diseño experimental con la cámara de prueba de sensores adaptada para material particulado. Se adaptaron dos entradas, una de ellas conectada a una bomba de vacío, la otra al suministro de partículas y aire limpio. Externamente, se diseñó un sistema de adquisición de datos en tiempo real

La bomba de vacío posee dos utilidades, la primera es realizar pruebas con bajas presiones y la segunda disminuir una fracción de la presión interna con el fin de facilitar el posterior ingreso de partículas a la cámara al abrir una válvula de recuperación. Dentro de la cámara se colocaron un mini-ventilador para facilitar la circulación de partículas y disminuir el efecto de deposición durante las pruebas, además del sensor de PM y un sensor BME280 para medir la humedad, presión y temperatura internas (Fig. 2(a)). El sistema de partículas consta de un circuito que permite ingresar a la cámara la muestra de partículas, o aire limpio a través de un filtro HEPA (Fig. 2(b)).
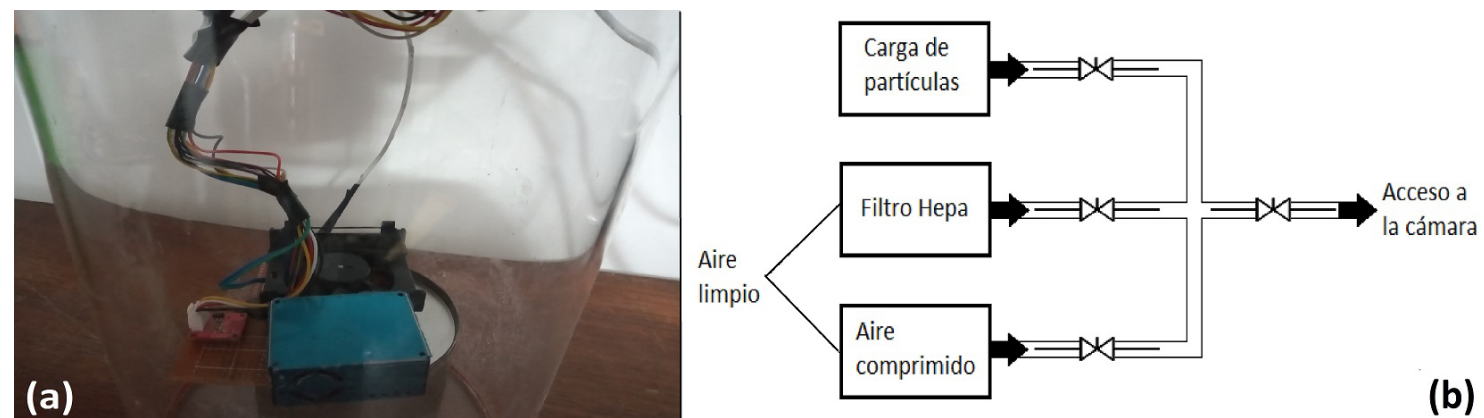

Fig. 2: Imagen más cercana de la cámara (a) y esquema del sistema de partículas (b). En el último se indican los circuitos para el ingreso de partículas, de aire limpio y la dirección de flujo hacia la cámara.

Los indicadores de desempeño evaluados en laboratorio para el sensor de PM se detallan a continuación:

- La precisión se determinó a partir de la Ec. (3): 


$$
P(\%)=100-\frac{E_{s}}{\bar{x}}
$$

Donde $\bar{x}$ es el valor promedio y Es es el error estándar del valor promedio de los sensores durante los períodos considerados como estado estable. El error estándar se calcula de acuerdo con la Ec. (4), donde x es cada valor medido y n la cantidad de mediciones (Polidori et al., 2016):

$$
E_{s}=\frac{\sqrt{\sum(x-\bar{x})^{2}}}{n}
$$

Se realizaron pruebas con aire sin filtrar para dos concentraciones de partículas diferentes, con medición continua del sensor dentro de la cámara sellada y con circulación interna de aire durante 15 minutos cada vez.

- Las mismas pruebas mencionadas en el ítem anterior se utilizaron para determinar la resolución, calculando las desviaciones estándar de las señales del sensor (Williams et al., 2014).

- Los tiempos de respuesta se evaluaron a partir de dos parámetros (Williams et al., 2014): - Tiempo de retraso: definido como el intervalo de tiempo entre un cambio en la variable y el primer cambio observable en la respuesta de medición. Ese cambio observable se considera como la tolerancia de precisión informada en la hoja de datos (PMS5003 datasheet, 2021). • Tiempo de subida: intervalo de tiempo entre la respuesta inicial y el $95 \%$ de la respuesta final después de un aumento en la variable.

Se realizaron dos pruebas ingresando a la cámara una cantidad de partículas de polvo (tiza) en una de ellas, y humo (sahumerio) en la otra, y luego retirando el sensor de la cámara mientras está funcionando hacia un espacio con concentraciones ambiente. Se midió también el tiempo de bajada, definido como el tiempo desde que el sensor se trasladó a concentraciones ambiente y el $95 \%$ de la respuesta de las señales a concentraciones ambiente.

- La respuesta a la falta de energía se consideró como el tiempo que tarda el sensor en entrar en régimen y retomar valores normales después de una pérdida de energía (Polidori et al., 2016). Se evaluó cortando la alimentación y reconectándola varias veces dentro de la cámara sellada con concentraciones de aire no filtrado.

- El límite inferior de detección se evaluó a partir del método de Kaiser y Specker (1956), el cual se basa en la Ec. (5). Para la prueba de laboratorio, se determinó el valor de $k$ como 1 (al no depender de la lectura de un instrumento de referencia en este caso) y se disminuyó la presión dentro de la cámara hasta $400 \mathrm{hPa}$ con el fin de conseguir una baja concentración de partículas. 


$$
L L O D=\frac{3^{*} D E}{k}
$$

La Ec. (5) describe la forma de calcular el límite inferior de detección del sensor considerando un período de medición de campo en conjunto con un instrumento de referencia (sensor calibrado). Se utiliza la desviación estándar (DE) de la señal del sensor cuando la referencia mide concentraciones menores a $1 \mu \mathrm{g} / \mathrm{m}^{3}$ y la pendiente $k$ de la regresión lineal entre las mediciones del sensor a evaluar y la referencia.

Luego, se procedió a realizar una comparación del sensor PMS con un equipo calibrado Open-Seneca (Open-Seneca, 2021) del programa "Cambike" de la Universidad de Cambridge llevado a cabo en Buenos Aires. Este equipo posee un sensor de PM SPS30 (SPS de ahora en más) del fabricante "Sensirion" que es de bajo costo, pero que fue calibrado con un equipo regulatorio de la Embajada de Estados Unidos en CABA, por lo que se utilizó como referencia para evaluar el desempeño del PMS. Con las mediciones de campo de 11 días de duración midiendo en simultáneo humedad, presión y temperatura, junto con los sensores PSM y del SPS en funcionamiento, se realizó una descripción comparativa y se evaluaron las características del PMS que se detallan a continuación:

- Linealidad: se evaluó trazando las salidas del PMS contra la referencia, utilizando la regresión de mínimos cuadrados. Se calculan los coeficientes de determinación lineal (R2) y la raíz del error cuadrático medio (RMSE), como una medida de varianza y sesgo en el comportamiento de los sensores, utilizando las regresiones lineales. Además, se practicaron análisis de varianzas (ANOVA), se verificaron las potencias de los ajustes y se graficaron los cuantiles condicionales.

- Exactitud: se utilizaron también las regresiones lineales a partir de la desviación estándar respecto a la referencia (RED) (Wang et al., 2015), como se define en la Ec. (6) donde $\bar{v}$ es el valor medio de la serie del instrumento de referencia (SPS).

$$
R E D=\frac{100 * D E}{\bar{v}}
$$

- Sesgo del sensor: basado en la razón entre las concentraciones del PMS y el SPS en función del tiempo (Ec. (7)) (Sayahi et al., 2019)

$$
\operatorname{sesgo}_{t}=\left(\frac{P M S_{t}}{S P S_{t}}-1\right) * 100
$$

Donde PMS $_{t}$ es la concentración de PM en función del tiempo del sensor PMS y SPS es $_{t}$ lo equivalente para el sensor de referencia. Con el fin de mostrar una gráfica menos ruidosa, se practicó una media móvil horaria (promedios horarios de los datosen ) con los resultados de los sesgos en el período muestral original de 1 minuto.

- Límite inferior de detección: calculado esta vez utilizando la Ec. (5) con el criterio de mediciones menores a $1 \mu \mathrm{g} / \mathrm{m}^{3}$ de la referencia y utilizando la pendiente de la regresión lineal entre las señales de ambos sensores. 
Con el fin de contar con una referencia en torno al efecto de la HR en las señales del sensor de PM, se utilizó la librería "opcsim" (Hagan y Kroll, 2020) para lenguaje Python que permite modelar a medida sensores de PM desde un enfoque físico, así como construir distribuciones de partículas en base a diferentes parámetros. El sensor PMS es un nefelómetro que mide la luz láser, de $680 \mathrm{~nm}+/-10 \mathrm{~nm}$, dispersada a un ángulo de $90^{\circ}$ (Sayahi et al., 2019). Dichas características fueron usadas para modelarlo. El sensor modelado (llamado "neph1") se calibró bajo condiciones lo más cercanas posibles a las usadas por el fabricante del PMS (PMS5003 datasheet, 2021), con distribuciones del tipo urbana y bajo porcentaje (50 \%) de HR. Luego, se calibra el mismo sensor modelado con una distribución de sulfato de amonio en solución y 0 \% de HR (llamado “neph2"). Esta solución se estableció con $\mathrm{N}=$ $1000, \bar{D}_{p}=0,08, \sigma=1,5, \mathrm{mi}=1,521+0 \mathrm{j}, \rho=1,77 \mathrm{~g} / \mathrm{cm}^{3} \mathrm{y} f(\mathrm{RH})=0,53$ (Hagan y Kroll, 2020). Luego, se calcularon los cambios en las distribuciones y carga másica de partículas medidas bajo diferentes condiciones de HR. Para comparar estos resultados con el comportamiento real del sensor PMS, se utilizó nuevamente la cámara de laboratorio para inyectar una solución de sulfato de amonio de concentración $10 \% \mathrm{~m} / \mathrm{V}$ con el sensor funcionando dentro bajo diferentes condiciones de HR ( 5 niveles de humedad controlados con un humidificador ultrasónico y partiendo de un nivel bajo en presencia de esferas de silicagel). Los niveles de HR conseguidos no son exactos por la variabilidad dentro de la cámara, pero sí están diferenciados entre sí y son de aproximadamente 20, 30, 50, 70 y $90 \%$.

Finalmente, con la información y resultados obtenidos de las pruebas de laboratorio, comparaciones con el SPS y simulaciones con la librería "opcsim", se establecieron criterios de gestión de calidad de los datos del PMS para ser evaluados de forma automática durante su funcionamiento. Esto se logró diseñando un algoritmo adosado al control del sensor que permite incorporar un dato más a la serie de la estación con un código que facilite al usuario identificar valores sospechosos o eventos puntuales, con marcadores que en este contexto suelen llamarse "banderas".

\section{Resultados}

La Tabla 1 resume los resultados de las pruebas de laboratorio:

\begin{tabular}{|c|c|c|c|}
\hline Indicador & $\mathrm{PM}_{1}$ & $\mathrm{PM}_{2.5}$ & $\mathrm{PM}_{10}$ \\
\hline Precisión $(\%)$ & $\sim 100$ & $\sim 100$ & $\sim 100$ \\
\hline Resolución $\left(\mu \mathrm{g} / \mathrm{m}^{3}\right)$ & 1,3 & 2,3 & 2,9 \\
\hline Tiempo de retraso (s) & $<1$ & $<1$ & $<1$ \\
\hline Tiempo de subida (s) & $20-57$ & $23-54$ & $30-57$ \\
\hline Tiempo de bajada (s) & $273-326$ & $273-325$ & $273-326$ \\
\hline LLOD $\left(\mu \mathrm{g} / \mathrm{m}^{3}\right)$ & 3,3 & 4,4 & 4,4 \\
\hline
\end{tabular}

Tabla 1: Indicadores de desempeño del sensor PMS determinados en las diferentes pruebas de laboratorio

Ambas pruebas con valores cuasi-estacionarios de concentraciones de partículas resultaron con errores estándar insignificantes y precisión cercana al 100\%. Respecto a las resoluciones 
para cada rango de tamaños, los valores son mayores mientras mayores sean las concentraciones medias. Se debe considerar que $\mathrm{PM}_{10}$ es una fracción poco confiable en los LCS ya que en la mayoría de los casos se calcula a partir de la fracción de $\mathrm{PM}_{2.5}$ (Alfano et al., 2020), esto es informado por el fabricante del SPS30 (ver Sensirion 2021) y no hemos encontrado hasta la fecha esta especificación para el PMS5003.

En cuanto a los tiempos de respuesta del sensor, las señales para la primera prueba con perturbación a partir de humo de sahumerio dentro de la cámara se muestran en la Fig. 3, y para la segunda prueba con polvo de tiza en la Fig. 4. Los tiempos de retraso (reacción a la perturbación) son insignificantes en todos los casos, o al menos menores a $1 \mathrm{~s}$ que es el período muestral utilizado. También se puede ver que el tiempo de subida (alcance del valor máximo medido) es muy similar en las señales de las tres fracciones de tamaños en la primera prueba (entre 54 s y 57 s) y un poco diferentes en la segunda prueba (entre 20 s y 30 s). Los tiempos de bajada (recuperación a valores ambiente) son notablemente mayores a los de subida, pues en la primera prueba son de alrededor de $273 \mathrm{~s}$, y de unos $325 \mathrm{~s}$ en promedio para la segunda. Esto implica que las partículas permanecen por un tiempo en la cámara de muestreo o en el circuito desde la toma de aire hasta la misma. Esto significa que el sensor puede tomarse entre $20 \mathrm{~s}$ y 1 minuto en alcanzar el valor que mide en un evento de aumento significativo de concentración, y más de 5 minutos en recuperar valores de ambiente luego de finalizado el mismo.

En la Fig. 4 se observa también un pico a los 320 s, el cual puede deberse a una re-suspensión de las partículas dentro de la cámara de sensado. Este fenómeno se observó en mayor o menor medida y en diferentes tiempos para todas las pruebas realizadas con polvo de tiza.
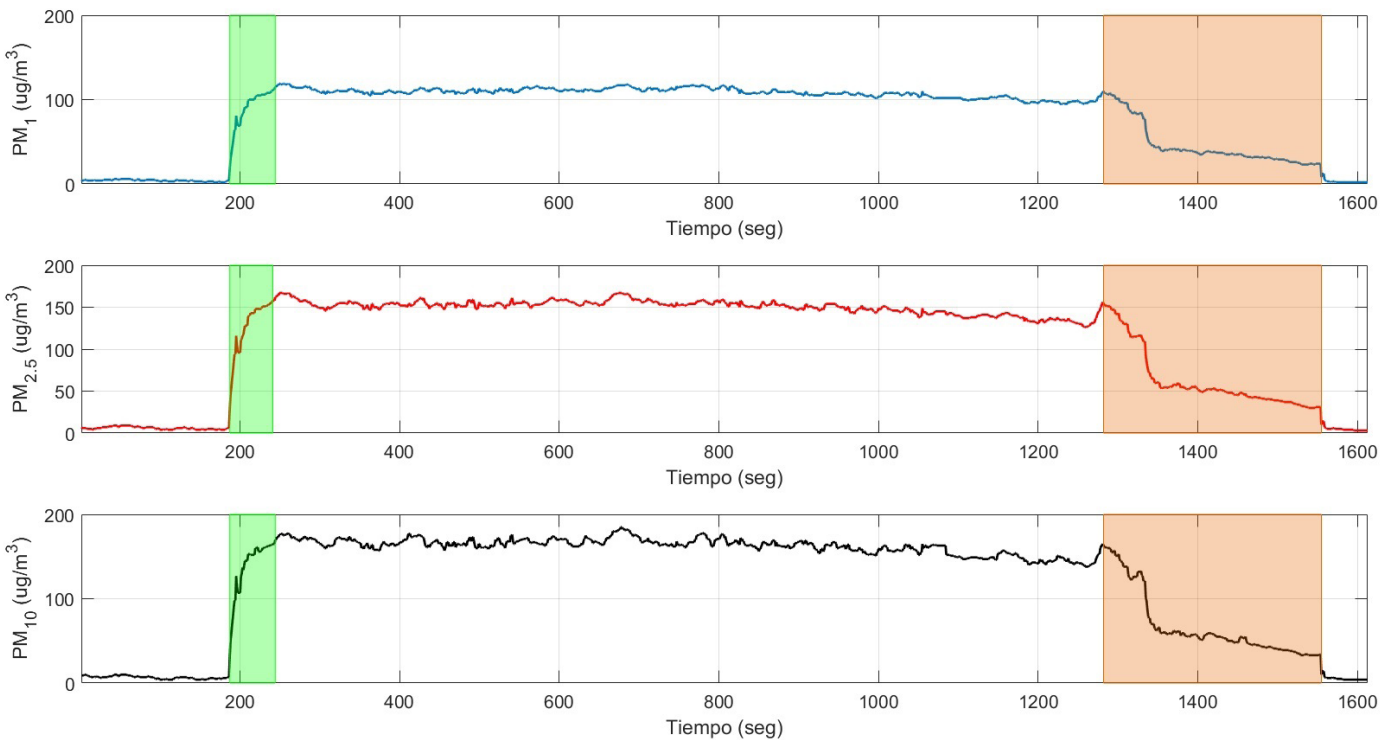

Fig. 3: Señales de PM del sensor para la prueba 1 de respuesta con humo de sahumerio en la cámara. El tiempo de subida está sombreado en verde, y el de bajada con color naranja 

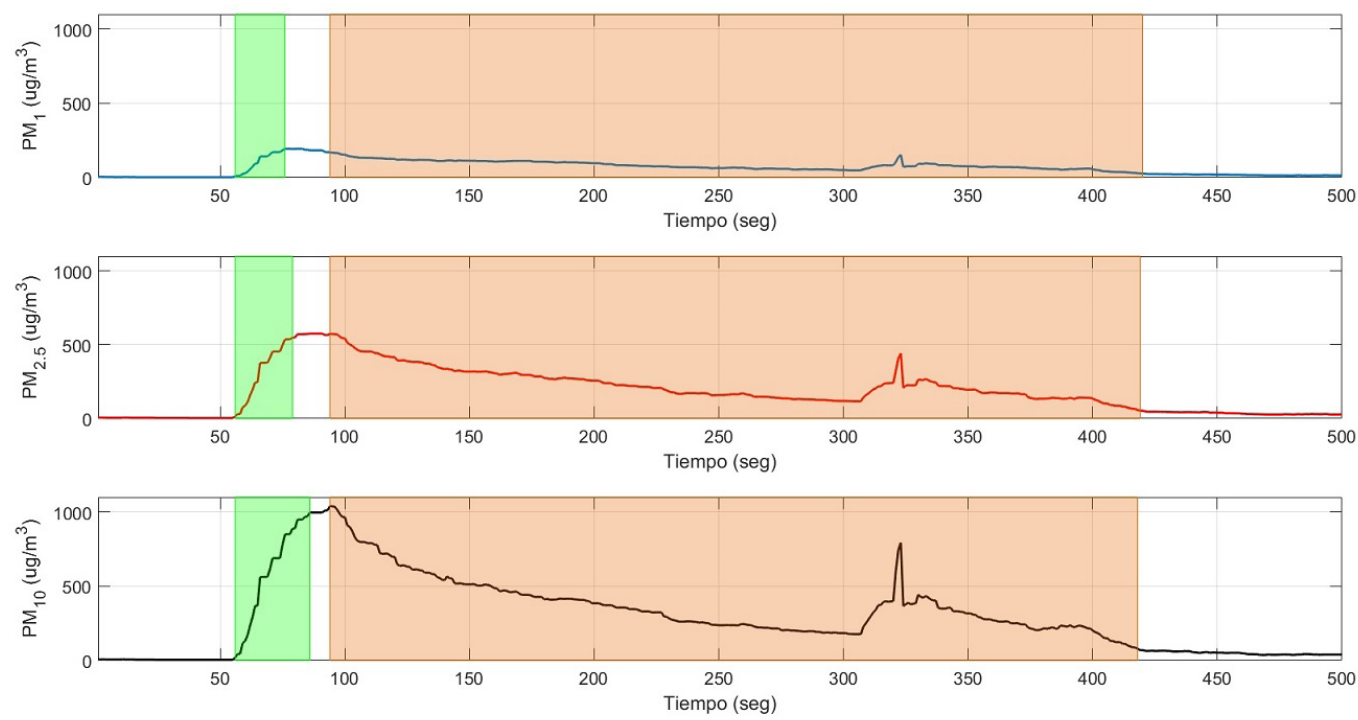

Fig. 4: Señales de PM del sensor para la prueba 2 de respuesta con polvo de tiza en la cámara. El tiempo de subida está sombreado en verde, y el de bajada con color naranja

Respecto a la respuesta a la falta de energía, no se registraron cambios en las señales del sensor entre los valores previos y posteriores a los cortes. Por último, en las pruebas realizadas disminuyendo la presión de la cámara se determinaron límites de detección superiores a $3 \mu \mathrm{g} / \mathrm{m}^{3}$ en todos los casos.

La Tabla 2 condensa los resultados de los indicadores obtenidos de la comparación para el PMS.

\begin{tabular}{|c|c|c|c|}
\hline Indicador & $\mathrm{PM}_{1}$ & $\mathrm{PM}_{2.5}$ & $\mathrm{PM}_{10}$ \\
\hline Linealidad $\left(\mathrm{R}^{2} / \mathrm{RMSE}\left(\mu \mathrm{g} / \mathrm{m}^{3}\right)\right)$ & $0,97 / 4,9$ & $0,97 / 8,7$ & $0,97 / 10,1$ \\
\hline Exactitud $(100 \%-\mathrm{RED})$ & 84,2 & 80,2 & 78,7 \\
\hline Sesgos $(\% / \mathrm{h})$ & 0,12 & 0,15 & 0,15 \\
\hline $\operatorname{LLOD}\left(\mu \mathrm{g} / \mathrm{m}^{3}\right)$ & 1,6 & 2,1 & 3,3 \\
\hline
\end{tabular}

Tabla 2: Indicadores de desempeño del sensor PMS en comparación con la referencia de SPS

Los cuantiles condicionales de los valores registrados por los sensores PMS y SPS en la campaña de comparación se muestran en la Fig. 5. El hecho de que la mediana (trazada en rojo) se encuentre siempre por debajo del ajuste perfecto (línea azul), indica que el PMS presenta sistemáticamente mayores valores de concentración que la referencia, para las tres señales. Los percentiles se encuentran en todo momento cercanos a la línea de la mediana, por lo que no hay demasiada dispersión al respecto. Esa diferencia se observa también en los histogramas, donde se aprecian distribuciones similares para todos los casos con un leve 
sesgo hacia la izquierda para el PMS, dado que los bines de rangos de mayor concentración tienen mayor conteo que para el SPS. Estos resultados muestran un error sistemático del sensor comparado con la referencia (con niveles de concentración mayores para el PMS en promedio de $3,2 \mu \mathrm{g} / \mathrm{m}^{3}$ para $\mathrm{PM}_{1}, 5,8 \mu \mathrm{g} / \mathrm{m}^{3}$ para $\mathrm{PM}_{2.5}$ y $6,8 \mu \mathrm{g} / \mathrm{m}^{3}$ para $\mathrm{PM}_{10}$ ).
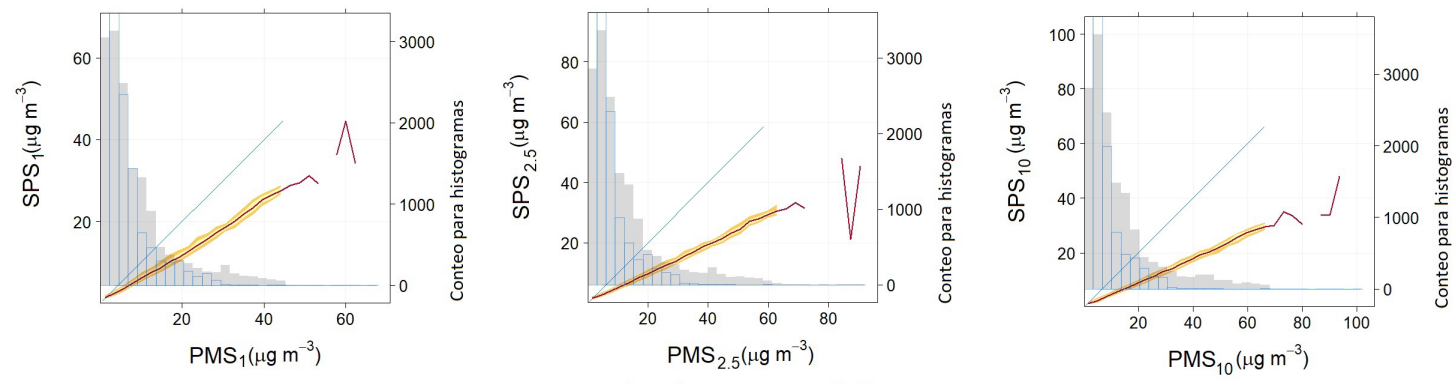

$\begin{array}{ll}\text { Percentiles } 25 / 75 & \text { Mediana } \\ \text { Percentiles } 10 / 90 & \text { Ajuste perfecto }\end{array}$

Fig. 5: Cuantiles condicionales entre las mediciones del equipo de referencia SPS (SPS, SPS $_{2.5}$ y SPS $_{10}$ ) y el PMS (PMS, PMS $_{2.5}$ y PMS $_{10}$ ) para las tres señales de PM en sus respectivos rangos de tamaños. Se trazan el ajuste perfecto (1:1), las medianas y percentiles del SPS en función de PMS y los histogramas (sombreado en gris para PMS y con línea azul para la referencia). La mediana presenta una porción aislada debido a un pico de concentración registrado durante las pruebas.

Respecto a los ajustes lineales, las rectas pasan cerca del origen, las pendientes también indican que las señales de PMS están siempre por encima de las del SPS y los coeficientes de correlación $(\mathrm{R})$ resultaron altos en todos los casos con un valor $\mathrm{p}$ del orden de $10^{-16}$. Además de estos indicadores de buen ajuste, la potencia de las regresiones indica que la certeza de las mismas es siempre cercana al $100 \%$. Luego, la desviación estándar relativa de PMS en torno a la referencia (RED) fue de 15,8 \% para los valores de $\mathrm{PM}_{1}, 19,8 \% \operatorname{para}^{\mathrm{PM}_{2.5}}$ y 21,3\% para $\mathrm{PM}_{10}$. La exactitud se calculó como la resta entre el 100\% y estos valores (Tabla 2).

Por otro lado, los sesgos del sensor en función del tiempo se muestran en la Fig. 6. Las pendientes mostradas en la Tabla 2 implican que la señal del sensor duplicaría el valor de sus residuales respecto a la referencia en casi un mes de funcionamiento continuo, aunque se requeriría de una campaña de comparación más extensa para aseverar este hallazgo.
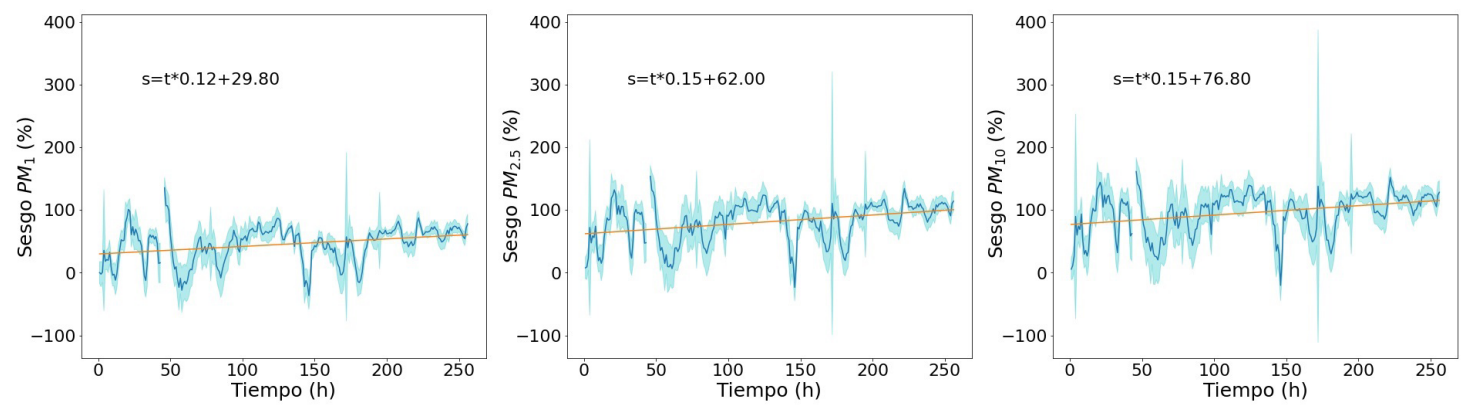

Fig. 6: Gráficas de los sesgos de las tres señales del sensor PMS en base a los residuales con la referencia SPS. En sombreado celeste se muestra la desviación estándar del promedio horario realizado y en naranja la recta de ajuste. También se muestran las ecuaciones del ajuste 
Los límites inferiores de detección (LLOD) fueron calculados nuevamente, pero con las pendientes de las regresiones y desviaciones estándar de PMS cuando el SPS midió menos de $1 \mu \mathrm{g} / \mathrm{m}^{3}$ (Tabla 2) y resultaron menores a los estimados con las pruebas de laboratorio.

Respecto a la comparación con sensores programados con la librería "opcsim”, la distribución de solución de sulfato de amonio simulada está compuesta de un sólo modo correspondiente a la fracción ultrafina, como muestra la Fig. 7. En la misma figura también se muestran los resultados de simulación del efecto de crecimiento higroscópico en la carga másica, donde la masa total crece un $800 \%$ entre los valores de humedad de $5 \%$ y $95 \%$.
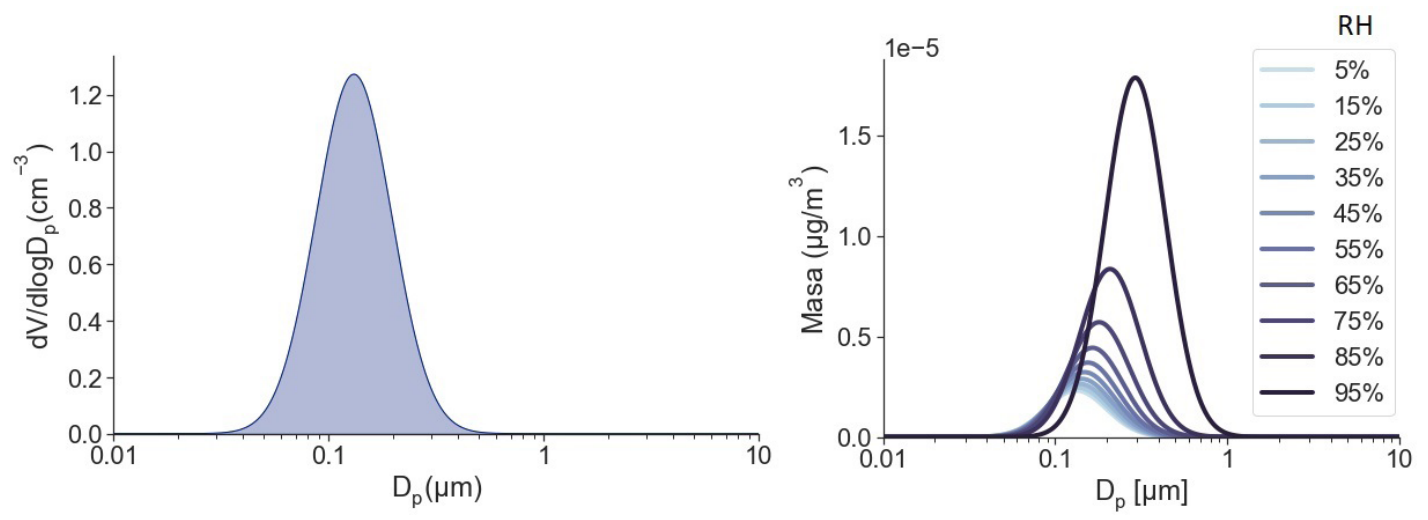

Fig. 7: Solución programada de sulfato de amonio. A la izquierda, distribución volumétrica. A la derecha, efectos de la HR en la carga másica de la solución por unidad de volumen. Los gráficos se trazaron en base a los resultados de las simulaciones.

Al compararse los efectos de la HR en las mediciones del sensor PMS con los de la distribución programada de sulfato de amonio y de los nefelómetros "neph1" y "neph2", lo primero que llama la atención es que se observan valores absolutos de las mediciones del sensor real (sensor PMS dentro de la cámara) en un orden de magnitud mayor que para las simulaciones, lo que es debido a las diferencias entre la solución simulada y las condiciones generadas dentro de la cámara real (Fig. 8), además de las propias incertezas del sensor para calcular la concentración de $\mathrm{PM}_{1}$. De todas formas, la comparación permite determinar que la solución ingresada a la cámara presenta un crecimiento higroscópico con la HR y que eso se traduce en una mayor masa acumulada, lo que es medido también por el sensor. Mientras que el crecimiento higroscópico dado por un aumento de HR desde el $20 \%$ al $90 \%$ se calculó en un $400 \%$ para la distribución modelada, la dispersión de la luz para los nefelómetros modelados aumentó en un 1200 \% (lo cual se tradujo en un similar porcentaje de aumento de masa en cada fracción de ambos). Es notable que el aumento en cada fracción de masa del sensor real fuese alrededor del $300 \%$, cercano a la de la distribución ideal, lo cual indica que la HR no parece afectar considerablemente su transducción, sino que el sensor logra correctamente medir las concentraciones dadas por el crecimiento higroscópico. Por otro lado, “neph1" simuló concentraciones más cercanas a la distribución ideal que "neph2", lo cual marca la importancia de calibrar estos sensores bajo las condiciones en las que serán utilizados. Según las simulaciones y pruebas realizadas, el crecimiento higroscópico comienza a afectar las mediciones del sensor con una HR a partir del 50 \%, y más considerablemente a partir del $70 \%$. 

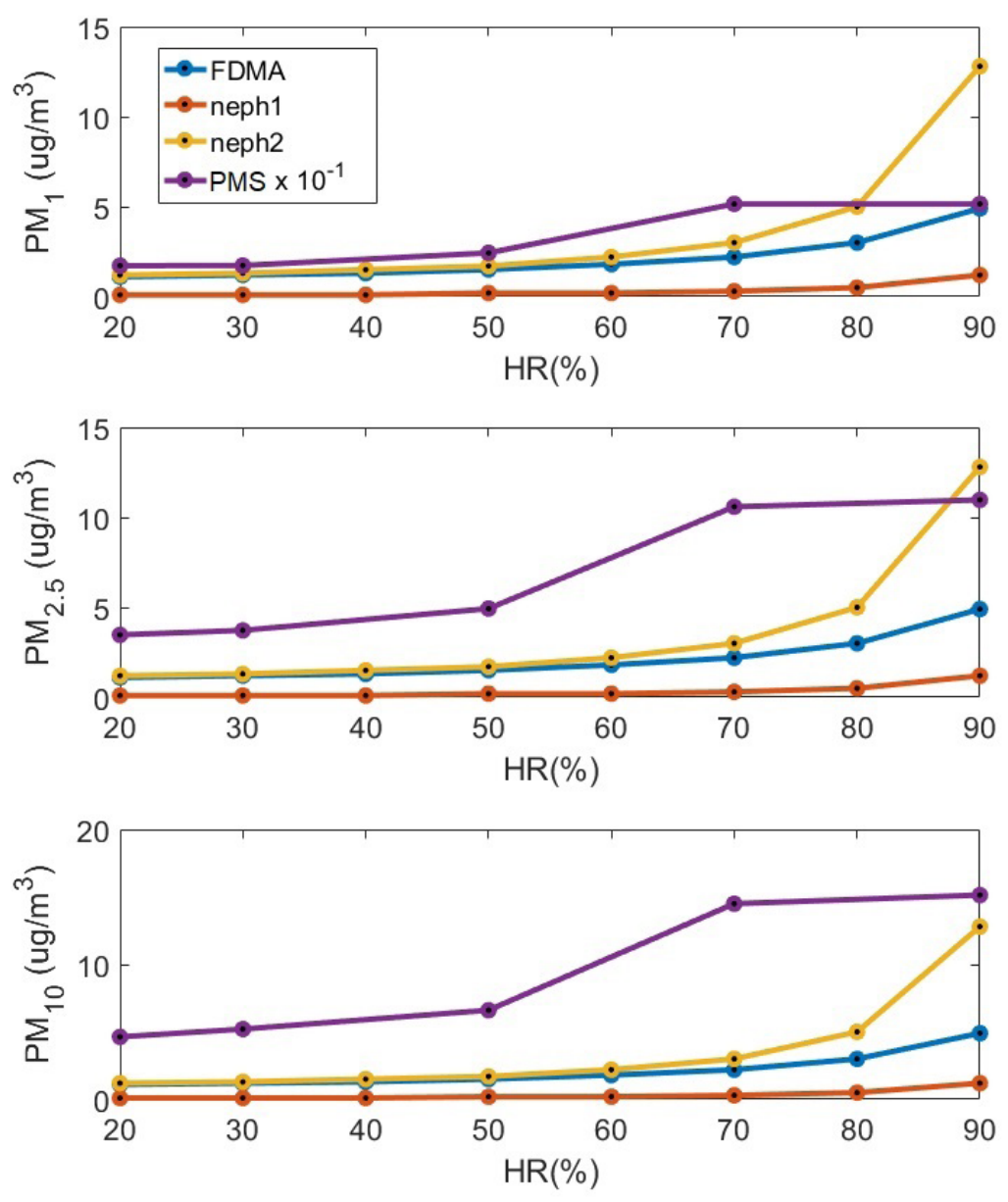

Figura 8. Efectos de la HR en simulaciones y en el sensor real PMS. Se muestran los valores de $\mathrm{PM}_{1}, \mathrm{PM}_{2.5} \mathrm{yPM}_{10}$ simulados para la solución de sulfato de amonio (FDMA) y para los nefelómetros programados (neph1 y neph2), junto con los medidos por el sensor en pruebas de laboratorio (marcado como PMS) con misma solución bajo distintas condiciones de HR. Para la solución simulada se muestra la función de distribución másica acumulada evaluada en cada rango de tamaños. Los valores de PMS fueron re-escalados para una mejor visualización

Los indicadores obtenidos de las pruebas muestran que el sensor PMS posee un buen rendimiento, considerando su bajo costo, como también se ha mencionado en otros antecedentes (Alfano et al., 2020). De todas formas, es necesario tener en cuenta sus limitaciones previo a su uso, por lo que se diseñó un ciclo dentro del algoritmo original de control que advierte al usuario sobre diferentes situaciones a través del código que se detalla en la Tabla 3. También cabe aclarar que se requieren de mayores pruebas y comparaciones para garantizar estos resultados en cuanto a valores absolutos. De todas formas, existen numerosas aplicaciones para los LCS donde se pone el foco en los valores relativos y en las tendencias 
de los datos adquiridos (por ejemplo, en mediciones orientadas a caracterizar fuentes de emisión).

\begin{tabular}{|c|c|}
\hline Notación & Advertencia \\
\hline A & Debajo del límite de detección inferior para $\mathrm{PM}_{1}\left(3,3 \mu \mathrm{g} / \mathrm{m}^{3}\right)$ \\
\hline B & Debajo del límite de detección inferior para $\mathrm{PM}_{2.5}\left(4,4 \mu \mathrm{g} / \mathrm{m}^{3}\right)$ \\
\hline C & Debajo del límite de detección inferior para $\mathrm{PM}_{10}\left(4,4 \mu \mathrm{g} / \mathrm{m}^{3}\right)$ \\
\hline D & Encima del límite de detección superior para $\mathrm{PM}_{2.5}\left(500 \mu \mathrm{g} / \mathrm{m}^{3}\right)$ \\
\hline $\mathbf{E}$ & Curva ascendente $\left(\mathrm{PM}^{\left.25 e c a_{t}-\mathrm{PM} 5 e c \mathrm{e}_{\mathrm{t}-1}>4,6 \mu \mathrm{g} / \mathrm{m}^{3}\right)}\right.$ \\
\hline $\mathbf{F}$ & Curva descendente $\left(\mathrm{PM}^{25 e c a_{\mathrm{t}}}-\mathrm{PM} 25 \mathrm{eca} \mathrm{t}_{\mathrm{t}-1}<-4,6 \mu \mathrm{g} / \mathrm{m}^{3}\right)$ \\
\hline G & $H R>50 \%$ \\
\hline H & HR $>70 \%$ \\
\hline
\end{tabular}

Tabla 3: Códigos utilizados para la gestión de calidad de datos. Los marcadores se incluyen en la línea de datos cuando se verifica alguna de las condiciones, y en el caso de ser más de una aparecerán consecutivas. Por ejemplo, una línea de datos marcada como BFH significa que el valor de $\mathrm{PM}_{25}$ se encuentra por debajo del límite inferior de detección, que las lecturas están en una curva descendente y que la HR está por encima del 70\%

Los límites inferiores de detección considerados son los informados en la Tabla 1, el límite superior para $\mathrm{PM}_{2.5}$ es el indicado en la hoja de datos del sensor (PMS5003 datasheet, 2021). La detección de curvas ascendentes y descendentes son útiles para que el usuario pueda considerar los tiempos de subida y bajada del sensor, y los criterios se establecen como una diferencia entre el valor actual y el anterior de $\mathrm{PM}_{2.5}$ mayor a dos veces la resolución de la Tabla 1 o menor a dos veces el valor negativo de la misma resolución, respectivamente. Finalmente, la HR tiene un efecto en las mediciones que debe ser tenida en cuenta, por ello se advierte cuando los valores superan el $50 \%$ y cuando se encuentran por encima del $70 \%$.

\section{Conclusiones}

Las pruebas de laboratorio, comparaciones con el equipo de Cambike y simulaciones con la librería "opcsim" arrojaron resultados que sirvieron para caracterizar al sensor de PM PMS5003. Este mostró una buena precisión y linealidad, así como un tiempo de respuesta casi inmediato y aceptables niveles de resolución y límites inferiores de detección (diferentes a los informados en la hoja de datos). Los tiempos de subida y bajada son un aspecto importante a considerar cuando se detectan episodios de corta duración, ya que el sensor puede demorarse hasta 1 minuto en alcanzar el valor real de medición y más de 5 minutos en alcanzar nuevamente los valores estables una vez finalizado el evento. Una preocupación puede ser el sesgo del sensor, una deriva que con el paso del tiempo en un funcionamiento continuo puede afectar las mediciones, aunque se necesita comparar con equipo bajo regulación para verificarlo. Por otro lado, las pruebas con las soluciones de sulfato de amonio demostraron que el sensor se ve afectado por la humedad relativa (HR), aunque al mismo nivel que las simulaciones siguiendo el crecimiento higroscópico de las partículas.

El código de gestión de calidad de datos desarrollado en base a los hallazgos en este 
estudio facilitará al usuario la detección de cualquier anomalía o condiciones de cuidado para las mediciones de PM. Este sensor se encuentra actualmente en funcionamiento en la zona y sus mediciones aportarán información a un nivel relativo que aun así permitirá elaborar estudios sin precedentes en la zona.

Resta para futuros trabajos realizar pruebas con controles más precisos de HR y también verificar su comportamiento en torno a la temperatura, además de utilizar soluciones en la cámara de calibración con otras fracciones de tamaño (más cercanas a $\mathrm{PM}_{2.5}$ ). Por otro lado, se utilizó una sola unidad del PMS5003 para su caracterización, por lo que estos resultados no pretenden reemplazar los de las hojas de datos o ser tomados con absolutos por otro usuario con otra unidad del sensor, pero la metodología presentada puede ser reproducida para tal fin.

\section{Agradecimientos}

Los autores agradecen a Norberto Vidal del Ministerio de Ambiente y Desarrollo Sostenible de la Nación por la vinculación en el contexto de las campañas de Cambike llevadas a cabo en Buenos Aires. Asimismo, se agradece a los integrantes del programa Open-Seneca por facilitar el equipo calibrado. 


\section{Referencias}

Alfano, B., Barretta, L., Del Giudice, A., De Vito, S., Di Francia, G., Esposito, E., ... \& Polichetti, T. (2020). "A review of low-cost particulate matter sensors from the developers' perspectives”. Sensors, 20(23), 6819.

AQ-SPEC. South Coast Air Quality Management District (2021). url: http://www.aqmd.gov/ aq-spec. Visitado el 15-10-2021.

Campbell, J. L., Rustad, L. E., Porter, J. H., Taylor, J. R., Dereszynski, E. W., Shanley, J. B., ... \& Boose, E. R. (2013). "Quantity is nothing without quality: Automated QA/QC for streaming environmental sensor data”. BioScience, 63(7), 574-585.

Chow, J. C., \& Watson, J. G. (1998). "Guideline on speciated particulate monitoring”. Report prepared for US Environmental Protection Agency, Research Triangle Park, NC, by Desert Research Institute, Reno, NV.

Crilley, L. R., Shaw, M., Pound, R., Kramer, L. J., Price, R., Young, S., ... \& Pope, F. D. (2018). "Evaluation of a low-cost optical particle counter (Alphasense OPC-N2) for ambient air monitoring”. Atmospheric Measurement Techniques, 11(2), 709-720.

Hagan, D. H., \& Kroll, J. H. (2020). "Assessing the accuracy of low-cost optical particle sensors using a physics-based approach". Atmospheric measurement techniques, 13(11), 63436355 .

Kaiser, H., \& Specker, H. (1956). "Bewertung und vergleich von analysenverfahren”. Fresenius’ Zeitschrift für analytische Chemie, 149(1), 46-66.

Karagulian, F., Barbiere, M., Kotsev, A., Spinelle, L., Gerboles, M., Lagler, F., ... \& Borowiak, A. (2019). "Review of the performance of low-cost sensors for air quality monitoring”. Atmosphere, 10(9), 506.

Kumar, P., Morawska, L., Martani, C., Biskos, G., Neophytou, M., Di Sabatino, S., ... \& Britter, R. (2015). "The rise of low-cost sensing for managing air pollution in cities”. Environment international, 75, 199-205.

Mie, G. (1976). "Contributions to the optics of turbid media, particularly of colloidal metal solutions”. Contributions to the optics of turbid media, 25(3), 377-445.

Open-Seneca. Open-Seneca organization. 2021. url: https://open-seneca.org/ (visitado 0706-2021).

Pascal, M., Corso, M., Chanel, O., Declercq, C., Badaloni, C., Cesaroni, G., ... \& Aphekom Group. (2013). "Assessing the public health impacts of urban air pollution in 25 European cities: results of the Aphekom project". Science of the Total Environment, 449, 390-400.

PMS5003 Datasheet (2021). url: https://www.aqmd.gov/docs/default-source/aq-spec/ resources-page/plantowerpms5003-manual_v2-3.pdf. Visitado el 04-06-2021.

Polidori, A., Papapostolou, V., \& Zhang, H. (2016). "Laboratory evaluation of low-cost air quality sensors". South Coast Air Quality Management District: Diamondbar, CA, USA.

Sayahi, T., Butterfield, A., \& Kelly, K. E. (2019). "Long-term field evaluation of the Plantower PMS low-cost particulate matter sensors". Environmental pollution, 245, 932-940. 
Scagliotti, A. F., \& Jorge, G. A. (2020a). "Análisis de un año de mediciones con fotómetro solar en el noroeste del conurbano bonaerense". ANALES AFA, 31(2), 46-50.

Scagliotti, A. F., \& Jorge, G. A. (2020b). "Inter-comparison of environmental low-cost sensors on Arduino platform”. Journal of the IEST, 63(1), 35-45.

Seinfeld, J. y Pandis S. "Atmospheric chemistry and physics: from air pollution to climate change”. John Wiley \& Sons, 2016.

Sensirion. Particulate Matter Sensor SPS30 (2021). url: https:/www.sensirion.com/en/ environmental-sensors/particulate-matter-sensors-pm25/. Visitado el 15-10-2021.

Taylor, K. E. (2001). "Summarizing multiple aspects of model performance in a single diagram”. Journal of Geophysical Research: Atmospheres, 106(D7), 7183-7192.

Tian, H., Qiu, P., Cheng, K., Gao, J., Lu, L., Liu, K., \& Liu, X. (2013). “Current status and future trends of SO2 and NOx pollution during the 12th FYP period in Guiyang city of China”. Atmospheric Environment, 69, 273-280.

Wang, Y., Li, J., Jing, H., Zhang, Q., Jiang, J., \& Biswas, P. (2015). “Laboratory evaluation and calibration of three low-cost particle sensors for particulate matter measurement”. Aerosol Science and Technology, 49(11), 1063-1077.

Williams, R., Long, R., Beaver, M., Kaufman, A., Zeiger, F., Heimbinder, M., ... \& Griswold, W. (2014). "Sensor evaluation report". US Environmental Protection Agency, Washington, DC, USA. 